\title{
Cognitive networks identify dimensions of distress in suicide notes: Anxiety, emotional profiles, and the "words not said"
}

\author{
Trevor James Swanson ${ }^{1 *}$, Andreia Sofia Teixeira ${ }^{2,3}$, Brianne N. Richson 1 , Ying Li 5 , Thomas T. Hills ${ }_{4}$ \\ Kelsie T. Forbush ${ }^{1}$, David Watson ${ }^{6}$ and Massimo Stella ${ }^{* 7}$
}

${ }^{1}$ Department of Psychology,

University of Kansas, 1415 Jayhawk

Blvd., Lawrence, KS, 66045, USA.

${ }^{2}$ Departamento de Inform 'atica,

Faculdade de $\mathrm{Ci}^{\wedge}$ encias, Universidade

de Lisboa, 1749-016 Lisboa, PT.

${ }^{3}$ LASIGE, Lisboa, PT.

${ }^{4}$ Department of Psychology,

University of Warwick, Coventry,

UK.

${ }^{5}$ Center for Adaptive Rationality,

Max Planck Institute for Human

Development, Berlin, DE.

${ }^{6}$ Department of Psychology,

University of Notre Dame,

Notre Dame, USA.

${ }^{7}$ CogNosco Lab, Department of

Computer Science, University of

Exeter, UK.

${ }^{*}$ Correspondence:

1 trevorswanson222@gmail.com

7 m.stella@exeter.ac.uk

\begin{abstract}
Suicide remains a serious public-health concern that is difficult to accurately predict in real-world settings. To identify potential predictors of suicide, we examined the emotional content of suicide notes using methods from cognitive network science. Specifically, we compared the co-occurrence networks of suicide notes with those constructed out of emotion words written by individuals scoring low or high on measures of depression, anxiety, and stress. Our objective was to identify which networks were most similar to the suicide notes network, in particular with regard to the connectivity between words and their emotional contents. We also investigated what types of words remained in the high/low emotion networks after controlling for the words present in the suicide notes, which we conceptualize as the "words not said" in the suicide notes. We found that patterns of connectivity among emotion words in suicide notes were most similar to those in texts written by low-anxiety individuals. However, upon analyzing the "words not said" in suicide notes, we observed that the remaining collection of emotions in suicide notes was most similar to those expressed by high-anxiety individuals. We discuss how these findings relate with existing clinical psychological literature as well as their potential implications for predicting suicidal behavior.
\end{abstract}

Keywords: complex networks; text analysis; emotional profiling; cognitive network science

\section{Introduction}

Approximately 800,000 people die by suicide every year - that is about one suicide every 40 seconds (World Health Organization, 2014). Suicide often arises from complex, distressed emotional processing that includes distorted perceptions about the self and others, as well the world in general (Oquendo et al., 2020; Rizk et al., 2019). Given the variety and complexity of factors that may lead an individual to contemplate or complete suicide, it is important to advance research into the psychological conditions surrounding such a tragic event. Suicide notes - that is, letters written immediately prior to the author's suicide - represent one potential window into the mindset of individuals who complete suicide (McAdams, 2001; Proulx \& Heine, 2006). By analyzing the contents and language of suicide notes, we can gain unique insights into the common features of individual experiences and obtain a greater understanding of the cognitive components that characterize suicidal ideation (Schneidman, 1981). 
Previous research on suicide notes has focused on identifying their contents, as well as what features of suicide notes differentiate them from other types of texts (Handelman \& Lester, 2007; Pestian et al., 2012b). Al-Mosaiwi \& Johnstone (2018) found that vocabulary of individuals at risk for suicide contained more absolutist words (e.g., "always", "totally", "entire") than what was observed among those diagnosed with depression or anxiety. Teixeira et al. (2021) found that suicide notes consisted of an overwhelming majority of positively-valenced words that exhibited a compartmentalized structure, such that positively- versus negatively-valenced words were often connected with one another (Showers, 1992). Moreover, Stella et al. (2021) found that suicide notes contained a higher degree of emotional complexity than notes written by a non-suicidal control group. Together, these findings reflect that suicide notes may contain a unique emotional and lexical footprint. However, an important question that remains is whether traces of that lexical footprint can be observed in texts written by individuals who exhibit high levels of emotional distress, such as stress, depression, and anxiety.

Several studies have shown that higher levels of stress, depression, and anxiety can be risk factors for suicidal ideation and behavior (Hawton et al., 2013; NaragonGainey \& Watson, 2011; Sandin et al., 1998; Sareen et al., 2005). Research has shown that among adults who reported a lifetime suicide attempt, up to $70 \%$ of them had an anxiety disorder (Nepon et al., 2010). Similarly, approximately $60 \%$ of individuals who completed suicide were diagnosed with major depressive disorder (Ng et al., 2017). Thus, to identify signs of suicidal ideation among individuals who report symptoms of internalizing disorders, it is an important goal to assess how the mindset of individuals who complete suicide might differ from the mindsets of individuals who exhibit psychopathological symptoms but did not attempt suicide.

In this paper we employ a cognitive network science framework to investigate the emotional contents and structure of suicide notes. To extend past research that used networks to study suicide notes (Stella et al., 2021; Teixeira et al., 2021), we aim to see how the emotional contents of such notes are related to those from a sample of texts written by individuals who reported high/low levels of stress, depression, and anxiety. The goal of this research is to shed light on the relationships between the mindsets of individuals experiencing these negative psychological conditions and the mindsets of individuals who completed suicide to inform future research on suicide prevention.

\section{Methods}

This section briefly outlines the datasets and network structures investigated in this study.

\section{Genuine suicide notes and ERT data}

This work used 139 genuine suicide notes curated by Schoene \& Dethlefs (2016) and investigated also by Teixeira et al. (2021). These letters were written in English by individuals who completed suicide. A suicide letter included an average of 120 words. No additional contextual information (e.g., demographics) was available from the dataset. Notes were processed with names and sensitive information anonymized by Schoene \& Dethlefs (2016), thus filtering out any sensitive information from the 
analysis. Text was tokenised (i.e., split into words) and stemmed (e.g., regularize different forms to a common morphonological root) through Mathematica 11.3. Punctuation was discarded and we considered only stemmed words appearing in the Emotional Recall Task (ERT).

The Emotional Recall Task was introduced by Li et al. (2020). $N=200$ individuals recruited from Amazon Mechanical Turk (MTurk) recalled 10 emotional states while reporting how they felt in their last month through a fluency task. The overall dataset included 475 different stems of emotional words (e.g., happy, sad, etc.) in English. We considered each recall as a list of words and stemmed it with Mathematica 11.3. Each word list/recall was relative to a numeric score from the Depression Anxiety Stress Scales (DASS) (Lovibond \& Lovibond, 1995). We partitioned ERT recalls as produced by people with higher-than-median and lower-than-median anxiety. We did the same for stress and depression, obtaining 6 different collections of ERT recalls.

To identify the structure of emotional co-occurrences across populations experiencing depression, anxiety, stress and suicide ideation, we used a cognitive network science framework (Gon i et al., 2011).

\section{Emotional co-occurrence networks}

We used the sequences of emotional words from suicide notes and ERT data to build networks of emotion co-occurrence in the narratives and recollections of our target populations. As in previous studies dealing with fluency data, we used the approach by Gon i et al. (2011) for building co-occurrence networks out of word lists. We used an $l=2$ window - i.e., we considered the two words that immediately preceded and followed each word in the texts as co-occurring together. We applied this window to all words in all sentences, and recorded all co-occurrences as connections between pairs of concepts. We then discarded all idiosyncratic co-occurrences, that is those appearing only once. We additionally selected links by considering a Binomial filtering based on word frequency in the corpora. All corpora featured a total of $|D|=475$ unique stems. These nodes $D$ were added to all networks, then connections for every emotional network were added according to the procedure by Gon $\sim$ i et al. (2011). This led to 7 emotional networks where nodes represent emotional states and links indicate stronger-than-expected emotional co-occurrences in clinical populations, i.e. people experiencing suicidal ideation and low/high depression, anxiety and stress.

\section{Emotional auras and Jaccard similarity}

Words in each emotional network were enriched with valence/arousal labels from the Valence-Arousal-Dominance dataset by Mohammad (2018). Labels for valence were "positive" (upper quartile), "negative" (lower quartile) or neutral (otherwise). Labels for arousal were "exciting" (upper quartile), "inhibitive" (lower quartile) or neutral (otherwise). These labels were used to detect the type of negative/positive or boring/exciting associations between emotional states. As in Stella et al. (2019), we used the metric of emotional auras to combine valence/arousal labels and network connectivity. For each word, we detected its neighborhood-i.e., the set of all words co-occurring with it. Example neighborhoods for "happi" are reported in 

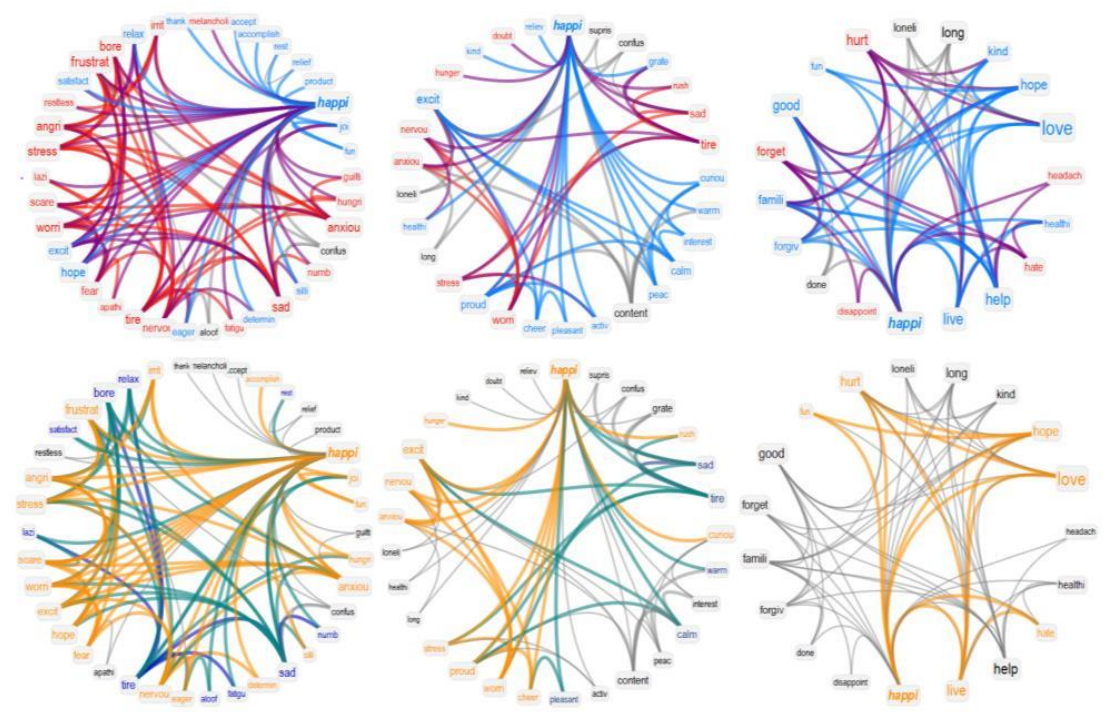

Figure 1 Semantic frame for "happi" (stem for "happiness", "happy" etc.) in the emotional networks by people with high depression (left), low depression (center) and suicide ideation (right), respectively. On the top (bottom) row, colours are based on valence (arousal). Positive (exciting) concepts are highlighted in cyan (orange) while negative (inhibiting) concepts are highlighted in red (teal). Neutral words are in black.

Figure 1. We then counted the most frequent valence-arousal polarities populating each neighborhood; this is the emotional aura attributed to a word and depending on its neighborhood of associated. The aura of each word does not depend on its valence-arousal labels, but rather on the affective labels of its neighbors/associates. For instance, "happi" (stem for "happiness" and "happy") is always labeled as a positive/arousing concept. However, it inherits a positive aura in the network produced by people with low depression, and a negative aura in the network produced by people with high depression, as represented in Figure 1. Disconnected nodes received neutral auras. By considering an ordered list of nodes $\left\{D^{1}, D^{2}, \ldots, D_{475}\right\}$, we represented each emotional network $\mathcal{N}$ as a vector:

$$
V^{a}(\mathcal{N})=\left\{A^{1}, A^{2}, \ldots, A_{475}\right\}
$$

of emotional auras for each corresponding node. By computing Jaccard similarity between vectors, we measured how similar emotional networks were in associating concepts with analogous emotional auras, mixing valence/arousal and emotion cooccurrence.

\section{Emotional profiling}

Emotional profiles were built by using the NRC Emotion Lexicon (Mohammad \& Turney, 2013). The latter is a behavioral mapping between words and the emotions elicited by them (Mohammad \& Turney, 2013).

Counting how many emotions are present in a given set powered our "words not said" analysis. We measured residual emotions present in the complement $C$ of 
two lists of words with degree $k \geq 1$, one list coming from a network of low/high negative states and one list coming from suicide notes. For example, this procedure makes it possible to consider the emotions persisting in words that were mentioned by people with high depression but not mentioned/associated in suicide notes.

For any complement list $C$, we reconstructed its affective content as an emotional flower (Semeraro et al., 2021; Stella, 2020), where petals indicate $z$-scores of eight different emotional states based on how many words elicit a given state. The larger the petals, the more concepts elicit that emotional state. Let us clarify this in quantitative terms. We measured the fraction $r_{i}=m / N$ of $m$ words inspiring emotion $i$ in a list with $N$ entries. The collection of observed values $\left\{r^{i}\right\}^{i}$ constitutes the so-called emotional profile of a set $C$ of words not said. For suicide notes, antonyms of words linked with meaning negations (e.g., "not") were added to the count (antonyms were defined via WordNet 3.0 (Fellbaum, 2012)). The observed profile was matched against 1000 random emotional profiles built by sampling uniformly at random from the NRC Emotion Lexicon as many words as those that elicited at least one emotion in the $\left\{r^{i}\right\}^{i}$ s. We computed $8 z$-scores, one per emotional state, and plotted them in a sector bar chart inspired to Plutchik's wheel of emotions (Plutchik, 1980). The rejection area $z<1.96$ was plotted as a semi-transparent area and concentric circles indicated units of $z$-scores higher than 2 .

\section{Results}

Suicide notes' semantic frames most resemble positive and arousing associations expressed by individuals with low anxiety

First, as described by Teixeira et al. (2021), we observed that the suicide notes contained an overwhelming majority of words with positive auras. Specifically, only 3 words were observed to have negative auras. Given the paucity of words with negative auras in the suicide notes as compared with the ERT data, we were not able to construct appropriate comparisons for words with negative auras across the two datasets. Thus, we limited the analysis of Jaccard similarity to words with positive auras. In addition to assessing the similarity of words with positive auras, we also included exciting auras as a second dimension for computing the Jaccard similarity with each ERT network against the suicide notes network.

The results for this analysis are displayed in Figure 1, with the Jaccard similarity of words with positive auras plotted on the y-axis, and that for words with exciting auras plotted on the x-axis. Each point on the plot represents the Jaccard similarity between each ERT network and the suicide notes network on each of the two dimensions. The first pattern that emerges is that low stress, anxiety, and depression networks have higher similarity values for words with positive auras than the corresponding high stress, anxiety, and depression networks. Notably, we see that the low anxiety network has the highest levels of Jaccard similarity on both dimensions. This means that words with positive auras and exciting auras in both the suicide notes and low anxiety networks show the most similar patterns of connectivity with other words, as compared to what we observe for the other ERT networks. 
Going beyond positivity: "Words not said", suicide letters and high anxiety

Given that the ERT networks contained a variety of words with negative auras which were not able to be included in the analysis (due to the dearth of words with negative auras in suicide notes), this begs the question of what emotional contents exist in those networks that were not expressed in the suicide notes. That is, our initial analysis was limited by restricting the ERT networks to only evaluate words that were also included in suicide notes. Thus, we wanted to evaluate the residual emotional contents expressed by the "words not said" in suicide notes, but were said by the individuals who completed the ERT. To do this, we constructed residual networks for the ERT data, wherein for each subsample (low/high stress, anxiety, and depression) we removed all words that were present in suicide notes and analyzed the emotional profiles for the remaining words in each network.

Figure 2 shows the emotional flowers for each of the six ERT residual networks, which reflect the "words not said" in suicide notes. In each emotional flower the petals reflect the prevalence, and statistical significance, of each individual emotion among the words in the corresponding residual network. Petals that extend beyond the semi-transparent circles indicate that the $z$-score for that emotion is greater than 2, or that it appeared with a significantly greater frequency than chance, given an alpha level of .05. The only ERT network that did not reveal any emotions occurring more frequently than chance was the high anxiety network.

Closing remarks: Low and high anxiety are important constructs to understand emotions (not) expressed in suicide notes

Our results reveal an interesting relationship between the emotional contents of suicide notes and those from emotional-recall data provided from a sample of healthy individuals. Specifically, we observed that the patterns of connectivity among emotion words with positive and exciting auras in suicide notes showed the greatest similarity with those in texts written by relatively low-anxiety individuals. This alone does not tell the whole story. In fact, we see that after removing words used in suicide notes from networks generated by individuals high/low in stress, anxiety, and depression, all networks - except those representing written texts from highanxiety individuals - contained additional emotional contents not present in suicide notes.

\section{Discussion and Conclusions}

The purpose of the current study was to compare the emotional content of words in 139 genuine suicide notes curated by Schoene \& Dethlefs (2016) to 200 healthy individuals who completed the Emotional Recall Task in which they recalled 10 emotional states while reporting how they felt in their last month through a fluency task. Our secondary goal was to identify how many emotions were present in a given set of "words not said" by measuring residual emotions from a network of low/high emotion states and one list coming from suicide notes. We observed that patterns of connectivity among emotional words in suicide notes were most similar to those in texts written by low-anxiety individuals. At the same time, after removing the bulk of positive associations, the remaining collection of emotions in suicide notes was most similar to states expressed by high-anxiety individuals. 

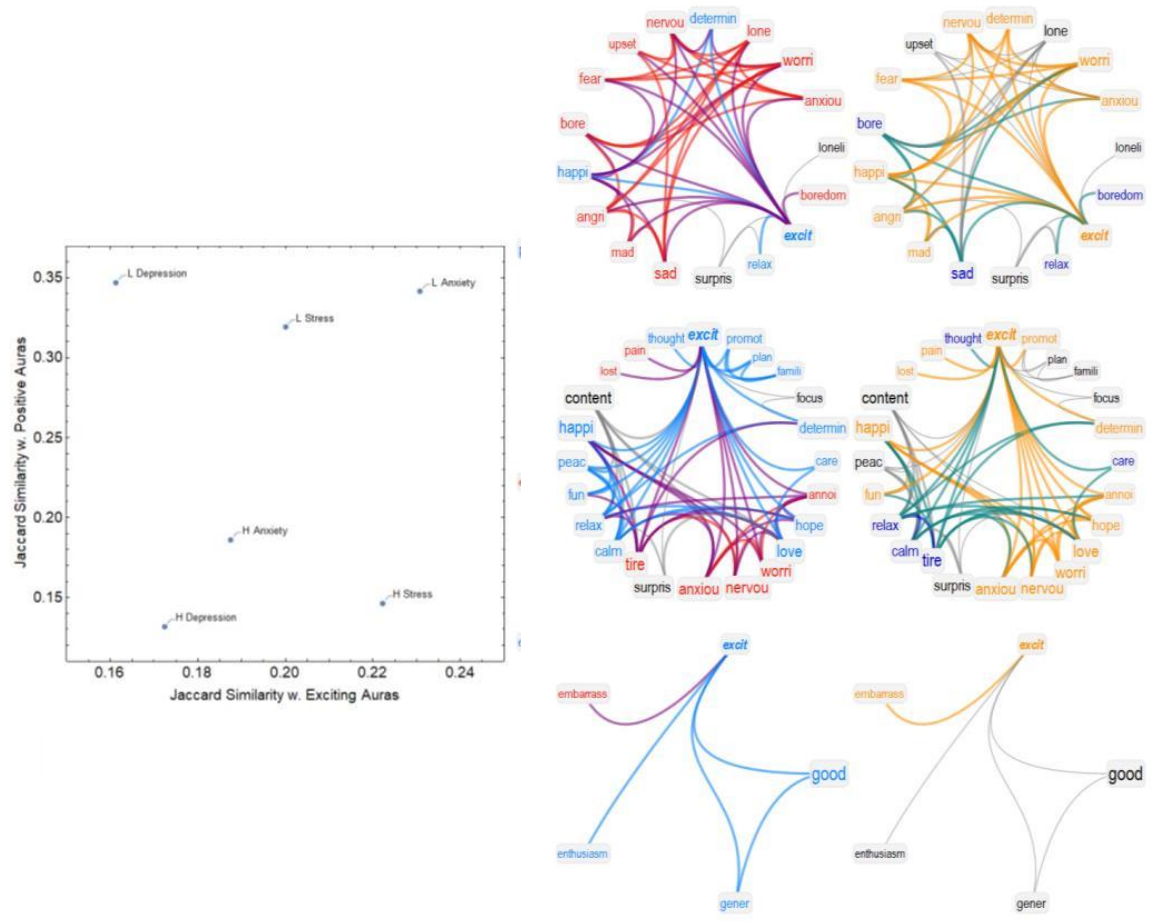

Figure 2 Right: Jaccard similarities between networks represented as vectors of emotional auras. Left: Semantic frame for "excit" (stem for "excitement", "exciting" etc.) in the emotional networks by people with high anxiety (top), low anxiety (center) and suicide ideation (bottom), respectively. On the left (right) column, colors are based on valence (arousal). Positive (exciting) concepts are highlighted in cyan (orange) while negative (inhibiting) concepts are highlighted in red (teal). Neutral words are in black.

Our findings reveal that the mindset of individuals who complete suicide has a complex association with the mindset of individuals experiencing anxiety. On first glance, it appears that the organization of emotion words in suicide notes is most similar to that in notes written by individuals with low levels of anxiety. But upon closer investigation, it seems that individuals with low anxiety also frequently express other types of emotions within their written texts, particularly a high level of joy and anticipation. However, heightened levels of these additional emotions appear to be absent among individuals who reported higher levels of anxiety, indicating that the collection of emotional contents they express matches more closely to that observed in suicide notes. An interesting conclusion of this research is that understanding how the emotional contents in suicide notes relate with emotions expressed by individuals with different levels of internalizing symptoms may have less to do with what is actually written, and more to do with the "words not said".

Suicidal ideation (a suicide risk factor) is associated with several anxiety-spectrum disorders and related disorders (e.g., post-traumatic stress disorder; NaragonGainey \& Watson, 2011). One meta-analysis indicated that the presence of an anxiety disorder distinguishes individuals who think about suicide from those who attempt suicide, whereas depression was similarly prevalent (May \& Klonsky, 2016). High-anxiety individuals may have an external locus of control (i.e., feeling one's life is controlled by external factors); perceived lack of control may facilitate feelings of hopelessness that, in theory, exacerbate suicidal ideation (Joiner, 2005). 


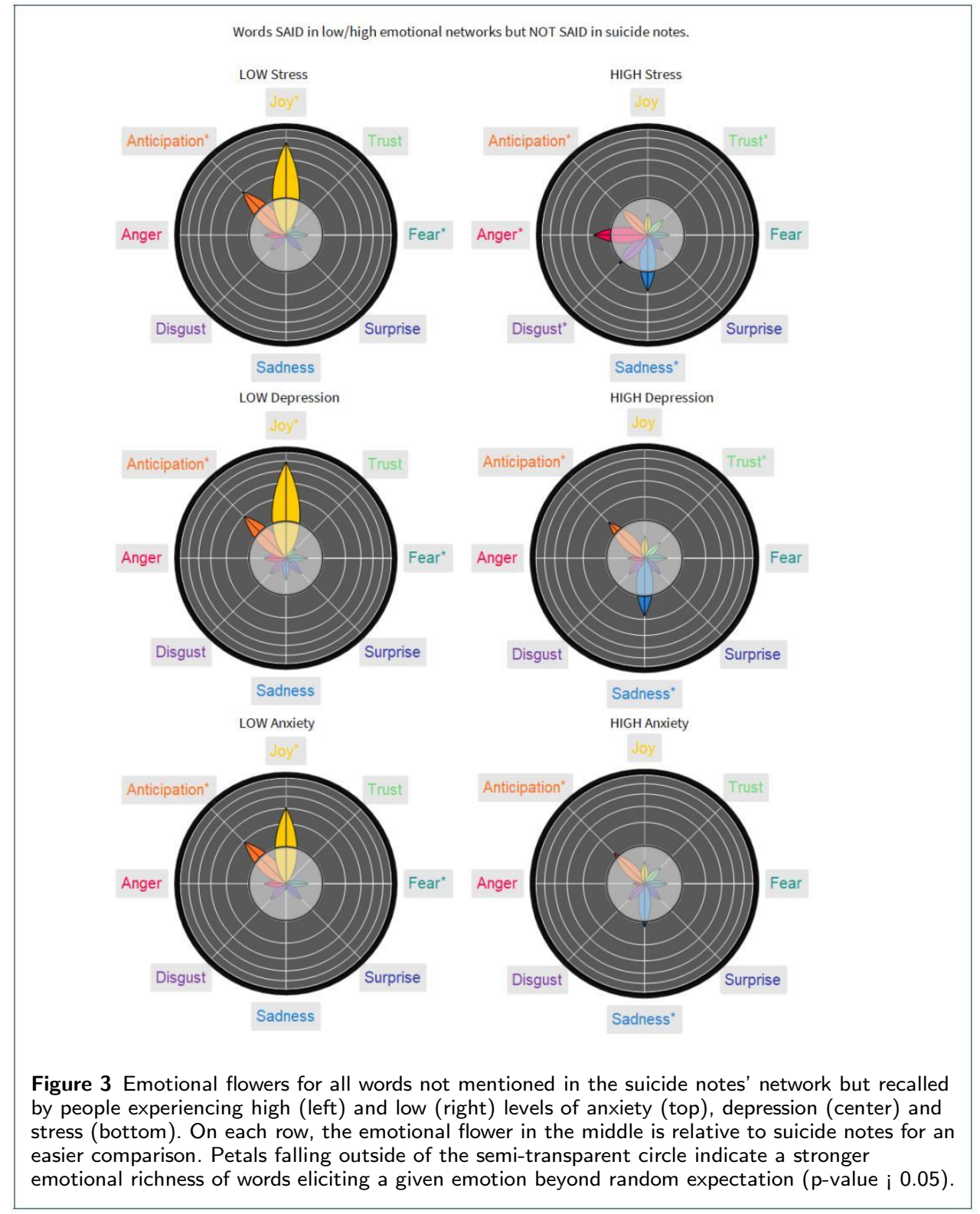

Indeed, having an external locus of control has been associated with suicide attempts (Wiebenga et al., 2021). It is also possible that 'high anxiety' is a proxy for overarousal and/or sensitivity to the experience of anxiety, both of which have been suggested as risk factors for suicide (Ribeiro et al., 2015; Stanley et al., 2018). More generally, neuroticism (a personality construct strongly related to anxiety) is likely negatively associated with positive affective experiences like joy $(\mathrm{Ng}, 2009)$.

The above provides some context to help explain why we observed a relationship between the emotions expressed by high-anxiety individuals and those expressed in suicide notes. However, the fact that patterns of connectivity among positivelyvalenced and exciting words in the suicide notes were most similar to those observed in low-anxiety individuals indicates that there is more to the story.

Multiple theories about suicide - e.g., the Interpersonal Theory of Suicide (Joiner et al., 2021), the 3-Step Theory (Klonsky \& May, 2015), and the Integrated 
Motivational-Volitional Model (O'Connor, 2011) - posit that there are distinct processes that lead one to develop suicidal ideation versus the capacity for acting on suicidal thoughts. In these theories, interpersonal factors such as loneliness or feeling as though one does not belong with their social group may lead to suicidal thoughts. However, the capacity for suicide appears to be related to certain aspects of fearlessness or low anxiety. This includes experiences such as low fear of bodily harm and a higher-than-average tolerance of physical pain (Joiner, 2005).

Thus our quantitative analysis reflects the dual nature of these relationships between anxiety and suicide, where there appears to be a contradictory association between both high/low anxiety and suicidal ideation/behavior. However, these associations may potentially be understood as reflecting what has been identified in past literature as a connection between higher emotional anxiety and greater risk of suicidal ideation (Naragon-Gainey \& Watson, 2011), as well as lower physiological anxiety being a risk factor for acting on suicidal thoughts (Joiner, 2005; Smith et al., 2010).

This distinction can be understood by looking closely at the items on the DASS "anxiety" subscale. This scale, which we used to operationalize high/low anxiety in our sample, contains mostly somatic and panic-related content (e.g., "I experienced difficulty breathing"; "I was aware of dryness of my mouth"), and a few anxious affect items (e.g., "I felt down-hearted and blue"). This may account for why we observed the most similarity between the emotional contexts of suicide texts and those written by individuals who scored low on the DASS anxiety subscale, given that a low anxiety score on the DASS may correspond more readily to low physiological anxiety than low emotional anxiety.

It is important to note some limitations of this study. First, there was no demographic information available for the authors of the suicide notes we analyzed in this study. The sample of individuals who completed the ERT was collected from MTurk (a population which has been extensively studied in the social science literature (Huff \& Tingley, 2015)), but it is unclear to what extent these individuals represented an appropriate comparison group for the sample of suicide-note authors. Future research would also benefit from analyzing a larger collection of suicide notes, such as the corpus of 1,319 suicide notes described by Pestian et al. (2012a) which includes relevant demographic information such as gender, age, and education level. This would likely help to address another limitation we encountered, which was the dearth of negatively-valenced words in the suicide notes.

Some strengths of this study lie in its potential implications for informing research on predicting suicidal behavior from written texts. Previous research has used machine learning techniques to identify texts that may signal suicidal ideation (Pestian et al., 2012b), but such methods often produce results that are difficult to understand and thus may not be very helpful for explaining the underlying features of a text that may signal risk for suicidal behavior (Rudin, 2019). Conversely, while qualitative methods for analyzing texts lend themselves better to interpretation, these may have limited reliability and be more difficult to apply on a broader scale (e.g., with larger corpora). Using cognitive network science, our approach retains the value of quantitative analysis while preserving the human dimension of the data. Network models allow us to analyze the patterns of associative knowledge 
that represent the mindsets of individuals who may be at risk for suicide. This affords interpretable, automated emotion detection which may be used to enhance clinical psychological investigations of an individual's risk for suicide.

Future research should clarify if similar results to what we observed here emerge in studies wherein depression, stress, and anxiety are modeled together, as we recognize that these constructs are not mutually exclusive and instead are highly related (O'Driscoll et al., 2021). In conclusion, results preliminarily suggest that text communication platforms could be monitored for textual-connectivity patterns, or a lack of key positive emotional language, to inform suicide prevention.

Competing interests

The authors declare that they have no competing interests.

Author's contributions

T.J.S., A.S.T., L.Y., T.T.H., and M.S. conceived and designed the study and interpreted the results. L.Y. and T.T.H. contributed the sample of ERT data. M.S. performed all numerical analyses and created all visualizations. B.N.R., K.T.F., D.W., and T.J.S. interpreted results in light of the relevant clinical psychological literature. All authors wrote and reviewed the manuscript.

Acknowledgements

This research has received no external funding.

Author details

${ }^{1}$ Department of Psychology, University of Kansas, 1415 Jayhawk Blvd., Lawrence, KS, 66045, USA.

${ }^{2}$ Departamento de Inform 'atica, Faculdade de Ci^encias, Universidade de Lisboa, 1749-016 Lisboa, PT. ${ }^{3}$ LASIGE, Lisboa, PT. ${ }^{4}$ Department of Psychology, University of Warwick, Coventry, UK. ${ }^{5}$ Center for Adaptive Rationality, Max Planck Institute for Human Development, Berlin, DE. ${ }^{6}$ Department of Psychology, University of Notre Dame, Notre Dame, USA. ${ }^{7}$ CogNosco Lab, Department of Computer Science, University of Exeter, UK.

\section{References}

Al-Mosaiwi, M. \& Johnstone, T. (2018). In an absolute state: Elevated use of absolutist words is a marker specific to anxiety, depression, and suicidal ideation. Clinical Psychological Science, 6(4), 529-542.

Fellbaum, C. (2012). WordNet. The Encyclopedia of Applied Linguistics.

Gon i, J., Arrondo, G., Sepulcre, J., Martincorena, I., De Mendiz'abal, N. V., Corominas-Murtra, B., Bejarano, B., Ardanza-Trevijano, S., Peraita, H., Wall, D. P., \& Villoslada, P. (2011). The semantic organization of the animal category: Evidence from semantic verbal fluency and network theory. Cognitive Processing, 12(2), $183-196$.

Handelman, L. D. \& Lester, D. (2007). The content of suicide notes from attempters and completers. Crisis, 28(2), 102-104.

Hawton, K., i Comabella, C. C., Haw, C., \& Saunders, K. (2013). Risk factors for suicide in individuals with depression: A systematic review. Journal of Affective Disorders, 147(1-3), 17-28.

Huff, C. \& Tingley, D. (2015). "Who are these people?" Evaluating the demographic characteristics and political preferences of MTurk survey respondents. Research \& Politics, 2(3), 2053168015604648.

Joiner, T. E. (2005). Why people die by suicide. Harvard University Press.

Joiner, T. E., Jeon, M. E., Lieberman, A., Janakiraman, R., Duffy, M. E., Gai, A. R., \& Dougherty, S. P. (2021). On prediction, refutation, and explanatory reach: A consideration of the interpersonal theory of suicidal behavior. Preventive Medicine, 152, 106453.

Klonsky, E. D. \& May, A. M. (2015). The three-step theory (3ST): A new theory of suicide rooted in the "ideation-to-action" framework. International Journal of Cognitive Therapy, 8(2), 114-129.

Li, Y., Masitah, A., \& Hills, T. T. (2020). The Emotional Recall Task: Juxtaposing recall and recognition-based affect scales. Journal of Experimental Psychology: Learning, Memory, and Cognition.

Lovibond, P. F. \& Lovibond, S. H. (1995). The structure of negative emotional states: Comparison of the Depression Anxiety Stress Scales (DASS) with the Beck Depression and Anxiety Inventories. Behaviour Research and Therapy, 33(3), 335-343.

May, A. M. \& Klonsky, E. D. (2016). What distinguishes suicide attempters from suicide ideators? A meta-analysis of potential factors. Clinical Psychology: Science and Practice, 23(1), 5.

McAdams, D. P. (2001). The psychology of life stories. Review of General Psychology, 5(2), 100-122.

Mohammad, S. (2018). Obtaining reliable human ratings of valence, arousal, and dominance for 20,000 english words. In Proceedings of the 56th Annual Meeting of the Association for Computational Linguistics (Volume 1: Long Papers) (pp. 174-184).

Mohammad, S. M. \& Turney, P. D. (2013). Crowdsourcing a word-emotion association lexicon. Computational Intelligence, 29(3), 436-465.

Naragon-Gainey, K. \& Watson, D. (2011). The anxiety disorders and suicidal ideation: Accounting for co-morbidity via underlying personality traits. Psychological Medicine, 41(7), 1437-1447.

Nepon, J., Belik, S.-L., Bolton, J., \& Sareen, J. (2010). The relationship between anxiety disorders and suicide attempts: Findings from the national epidemiologic survey on alcohol and related conditions. Depression and Anxiety, 27(9), 791-798. 
Ng, C. W. M., How, C. H., \& Ng, Y. P. (2017). Depression in primary care: Assessing suicide risk. Singapore Medical Journal, 58(2), 72.

$\mathrm{Ng}, \mathrm{W}$. (2009). Clarifying the relation between neuroticism and positive emotions. Personality and Individual Differences, 47(1), 69-72.

Oquendo, M. A., Galfalvy, H. C., Choo, T.-H., Kandlur, R., Burke, A. K., Sublette, M. E., Miller, J. M., Mann, J. J., \& Stanley, B. H. (2020). Highly variable suicidal ideation: A phenotypic marker for stress induced suicide risk. Molecular Psychiatry, (pp. 1-8).

O'Connor, R. C. (2011). Towards an integrated motivational-volitional model of suicidal behaviour. International Handbook of Suicide Prevention: Research, Policy and Practice, 1, 181-98.

O'Driscoll, C., Buckman, J. E., Fried, E. I., Saunders, R., Cohen, Z. D., Ambler, G., DeRubeis, R. J., Gilbody, S., Hollon, S. D., Kendrick, T., Kessler, D., Lewis, G., Watkins, E., Wiles, N., \& Pilling, S. (2021). The importance of transdiagnostic symptom level assessment to understanding prognosis for depressed adults: Analysis of data from six randomised control trials. BMC medicine, 19(1), 1-14.

Pestian, J. P., Matykiewicz, P., \& Linn-Gust, M. (2012a). What's in a note: Construction of a suicide note corpus. Biomedical Informatics Insights, 5, BII-S10213.

Pestian, J. P., Matykiewicz, P., Linn-Gust, M., South, B., Uzuner, O., Wiebe, J., Cohen, K. B., Hurdle, J., \& Brew C. (2012b). Sentiment analysis of suicide notes: A shared task. Biomedical Informatics Insights, 5, BII-S9042.

Plutchik, R. (1980). Emotion. A Psychoevolutionary Synthesis.

Proulx, T. \& Heine, S. J. (2006). Death and black diamonds: Meaning, mortality, and the meaning maintenance model. Psychological Inquiry, 17(4), 309-318.

Ribeiro, J. D., Yen, S., Joiner, T., \& Siegler, I. C. (2015). Capability for suicide interacts with states of heightened arousal to predict death by suicide beyond the effects of depression and hopelessness. Journal of Affective Disorders, 188, 53-59.

Rizk, M. M., Choo, T.-H., Galfalvy, H., Biggs, E., Brodsky, B. S., Oquendo, M. A., Mann, J. J., \& Stanley, B. (2019). Variability in suicidal ideation is associated with affective instability in suicide attempters with borderline personality disorder. Psychiatry, 82(2), 173-178.

Rudin, C. (2019). Stop explaining black box machine learning models for high stakes decisions and use interpretable models instead. Nature Machine Intelligence, 1(5), 206-215.

Sandin, B., Chorot, P., Santed, M. A., Valiente, R. M., \& Joiner Jr, T. E. (1998). Negative life events and adolescent suicidal behavior: A critical analysis from the stress process perspective. Journal of Adolescence, $21(4), 415-426$.

Sareen, J., Cox, B. J., Afifi, T. O., de Graaf, R., Asmundson, G. J., Ten Have, M., \& Stein, M. B. (2005). Anxiety disorders and risk for suicidal ideation and suicide attempts: A population-based longitudinal study of adults. Archives of General Psychiatry, 62(11), 1249-1257.

Schneidman, E. S. (1981). Suicide notes and tragic lives. Suicide and Life-Threatening Behavior.

Schoene, A. M. \& Dethlefs, N. (2016). Automatic identification of suicide notes from linguistic and sentiment features. In Proceedings of the 10th SIGHUM Workshop on Language Technology for Cultural Heritage, Social Sciences, and Humanities (pp. 128-133).

Semeraro, A., Vilella, S., \& Ruffo, G. (2021). Pyplutchik: Visualising and comparing emotion-annotated corpora. PLOS ONE.

Showers, C. (1992). Compartmentalization of positive and negative self-knowledge: Keeping bad apples out of the bunch. Journal of Personality and Social Psychology, 62(6), 1036

Smith, P. N., Cukrowicz, K. C., Poindexter, E. K., Hobson, V., \& Cohen, L. M. (2010). The acquired capability for suicide: A comparison of suicide attempters, suicide ideators, and non-suicidal controls. Depression and Anxiety, 27(9), 871-877.

Stanley, I. H., Boffa, J. W., Rogers, M. L., Hom, M. A., Albanese, B. J., Chu, C., Capron, D. W., Schmidt, N. B., \& Joiner, T. E. (2018). Anxiety sensitivity and suicidal ideation/suicide risk: A meta-analysis. Journal of Consulting and Clinical Psychology, 86(11), 946.

Stella, M. (2020). Text-mining forma mentis networks reconstruct public perception of the stem gender gap in social media. PeerJ Computer Science, 6, e295.

Stella, M., De Nigris, S., Aloric, A., \& Siew, C. S. (2019). Forma mentis networks quantify crucial differences in stem perception between students and experts. PLOS ONE, 14(10), e0222870.

Stella, M., Swanson, T. J., Li, Y., Hills, T. T., \& Teixeira, S. A. (2021). Cognitive network science as a framework for detecting suicidal patterns and emotions in suicide letters. PsyArxiv. https://doi.org/10.31234/osf.io/frvta.

Teixeira, A. S., Talaga, S., Swanson, T. J., \& Stella, M. (2021). Revealing semantic and emotional structure of suicide notes with cognitive network science. Scientific Reports, 11(1), 1-15.

Wiebenga, J. X., Eikelenboom, M., Heering, H. D., van Oppen, P., \& Penninx, B. W. (2021). Suicide ideation versus suicide attempt: Examining overlapping and differential determinants in a large cohort of patients with depression and/or anxiety. Australian \& New Zealand Journal of Psychiatry, 55(2), 167-179.

World Health Organization (2014). Preventing Suicide: A Global Imperative. World Health Organization, Geneva, Switzerland. 